\title{
COVID-19 por SARS-CoV-2: la nueva emergencia de salud
}

\author{
Ranferi Aragón-Nogales, ${ }^{1}$ Iván Vargas-Almanza, ${ }^{1}$ María Guadalupe Miranda-Novales $2, *$ \\ ${ }^{1}$ Residente de Infectología Pediátrica. UMAE, Hospital de Pediatría "Dr. Silvestre Frenk Freund”, Centro Médico Nacional \\ Siglo XXI; ${ }^{2}$ Unidad de Investigación en Análisis y Síntesis de la Evidencia, Coordinación de Investigación en Salud, Instituto \\ Mexicano del Seguro Social, México; Miembro del Plan Universitario para el Control de la Resistencia Antimicrobiana, UNAM. \\ Ciudad de México.
}

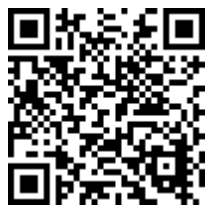

\section{INTRODUCCIÓN}

Los coronavirus se encuentran ampliamente distribuidos e infectan humanos, mamíferos y aves, pueden ocasionar enfermedades respiratorias, entéricas, hepáticas y neurológicas. De acuerdo con el Comité Internacional de Taxonomía de Virus, pertenecen al orden Nidovirales, familia Coronaviridae, subfamilia Coronavirinae, esta última consta de cuatro géneros Alphacoronavirus, Betacoronavirus, Gammacoronavirus y Deltacoronavirus. ${ }^{1}$ Se denominan coronavirus por la apariencia que dan bajo el microscopio electrónico parecido a una corona. Son virus envueltos, con un diámetro aproximado de $125 \mathrm{~nm}$, genoma ARN de cadena simple, sentido positivo. Se considera el genoma más grande de los virus ARN con un tamaño de 26-32 kilobases, codifica cuatro proteínas estructurales que incluyen glicoproteína espiga (S), envoltura (E), membrana (M) y nucleocápside $(\mathrm{N})$ y otras 16 proteínas no estructurales que participan en la transcripción y replicación viral como es la helicasa y la ARN polimerasa dependiente de ARN. ${ }^{2,3}$ Sobre la base de secuencias genéticas se sabe que todos los coronavirus humanos probablemente tienen un ancestro común, usan reservorios naturales o intermediarios en animales y tienen la capacidad de cruzar la barrera entre especies. ${ }^{4}$

\footnotetext{
*Correspondencia: MGMN, mmirandanovales@gmail.com Conflicto de intereses: Los autores declaran que no tienen. Citar como: Aragón-Nogales R, Vargas-Almanza I, Miranda-Novales MG. COVID-19 por SARS-CoV-2: la nueva emergencia de salud. Rev Mex Pediatr 2019; 86(6):213-218. doi: 10.35366/91871 [COVID-19 by SARS-CoV-2: the new health emergency]
}

Los coronavirus humanos a través de su proteína espiga se unen a uno de los receptores, éstos pueden ser: la enzima convertidora de angiotensina, dipeptidil peptidasa 4, aminopeptidasa $\mathrm{N}$ y $\mathrm{O}$-acidoacetil siálico, e ingresan a la célula a través de una vía endosómica y/o no endosómica; una vez ingresado, se libera la nucleocápside y el ARN en el citoplasma, se sintetizan las enzimas que participan en la transcripción y replicación del virus, se producen copias ARN de sentido negativo, por medio de ARN subgenómicos se producen las proteínas estructurales que posteriormente serán ensambladas y se libera el virión a través de exocitosis al espacio extracelular. ${ }^{5}$

El HCoV-229E, HCoV-OC43, HCoV-NL63 y $\mathrm{HCoV}-\mathrm{HKU} 1$ están distribuidos en todo el mundo, fueron identificados en 1966, 1967, 2004 y 2005, respectivamente, se transmiten sobre todo durante el invierno, son causa frecuente de resfriado común, con síntomas leves en pacientes inmunocompetentes. Existen dos virus altamente patógenos y transmisibles, el primero reportado en 2002 en la provincia de Guangdong, China, nombrado coronavirus del síndrome respiratorio agudo severo (SARS-CoV) y una década después en 2012 en Arabia Saudita se aisló el coronavirus del síndrome respiratorio del Medio Oriente (MERS-CoV). ${ }^{2,4}$

Los coronavirus despertaron el interés de la investigación médica al demostrar su capacidad epidémica. En 2003 se reportaron más de 8,098 casos de infección por SARS-CoV, con 916 muertes que correspondieron a una letalidad de $10 \%$ aproximadamente, catalogada como la primera nueva enfermedad infecciosa del siglo XXI que afectó a 29 países. En 2012, MERS-CoV infectó 
a 2,254 personas con 800 muertes, demostrando una alta mortalidad de $35 \%$ en 27 países afectados. ${ }^{3}$

\section{Nuevo coronavirus 2019}

Posterior a las epidemias de SARS-CoV y MERS-CoV, en China se establecieron estrategias para la identificación oportuna de virus emergentes y reemergentes. La vigilancia incluye la investigación y seguimiento de los casos de neumonía de etiología desconocida, así las neumonías que cumplan con los siguientes criterios: fiebre mayor de $38{ }^{\circ} \mathrm{C}$, recuento total de leucocitos normal o bajo, o recuento de linfocitos bajo, evidencia radiográfica de neumonía y no presentar mejoría en los síntomas después de tratamiento antimicrobiano por tres a cinco días, son sujetas a escrutinio. ${ }^{6} \mathrm{~A}$ finales de diciembre de 2019 en la ciudad de Wuhan, provincia Hubei, China, se reportaron una serie de casos que cumplían criterios para neumonía de etiología desconocida de características graves y las autoridades locales de salud notaron en los pacientes una asociación epidemiológica con un mercado mayorista de mariscos, en los que también se vendían al público animales no acuáticos como aves de corral vivas y varios tipos de animales salvajes. De forma inmediata notificaron al Centro Chino para el Control y Prevención de Enfermedades y el 31 de diciembre se inició la investigación epidemiológica y como primera medida de control el 01 de enero de 2020 se indicó el cierre del mercado al público. ${ }^{6-8}$
Las investigaciones iniciales de Zhu y colaboradores en muestras de lavado broncoalveolar de tres pacientes mediante el uso de reacción en cadena de la polimerasa en tiempo real (RT-PCR) y cultivo viral identificaron un nuevo virus, provisionalmente se nombró "nuevo coronavirus 2019" (nCoV-2019), el cual de acuerdo a sus características genómicas se clasificó dentro del género Betacoronavirus, subgénero Sarbecovirus y también se confirmaron sus efectos citopáticos con cambios estructurales en las células huésped. ${ }^{7}$ En el mercado de Wuhan se encontraron 15 muestras ambientales positivas para $\mathrm{nCoV}-2019 .{ }^{8}$ Un estudio publicado recientemente analizó el genoma completo del virus aislado de cinco pacientes al inicio del brote, y concluyeron que nCoV-19 es $96 \%$ idéntico al del genoma completo de un coronavirus de murciélago. También confirmaron que utiliza el receptor celular de la enzima convertidora de angiotensina (ACE2) y no los otros receptores. ${ }^{9}$

El 09 de enero el Centro Chino de los CDC informó el descubrimiento del nuevo coronavirus y el 12 de enero dieron a conocer la secuencia genómica del nCoV-2019 para el reporte de nuevos casos en otras regiones y el desarrollo de pruebas diagnósticas. ${ }^{8}$ De inmediato se observó un incremento exponencial en los casos confirmados. En un inicio la tasa de crecimiento epidémico fue de 0.10 por día (IC $95 \% 0.050$ a 0.16 ) y el tiempo de duplicación fue de 7.4 días (IC del 95\%, 4.2 a 14). ${ }^{6} \mathrm{El} 11$ de enero se reportó la primera defunción en China. El 13 de enero en Tailandia se registró

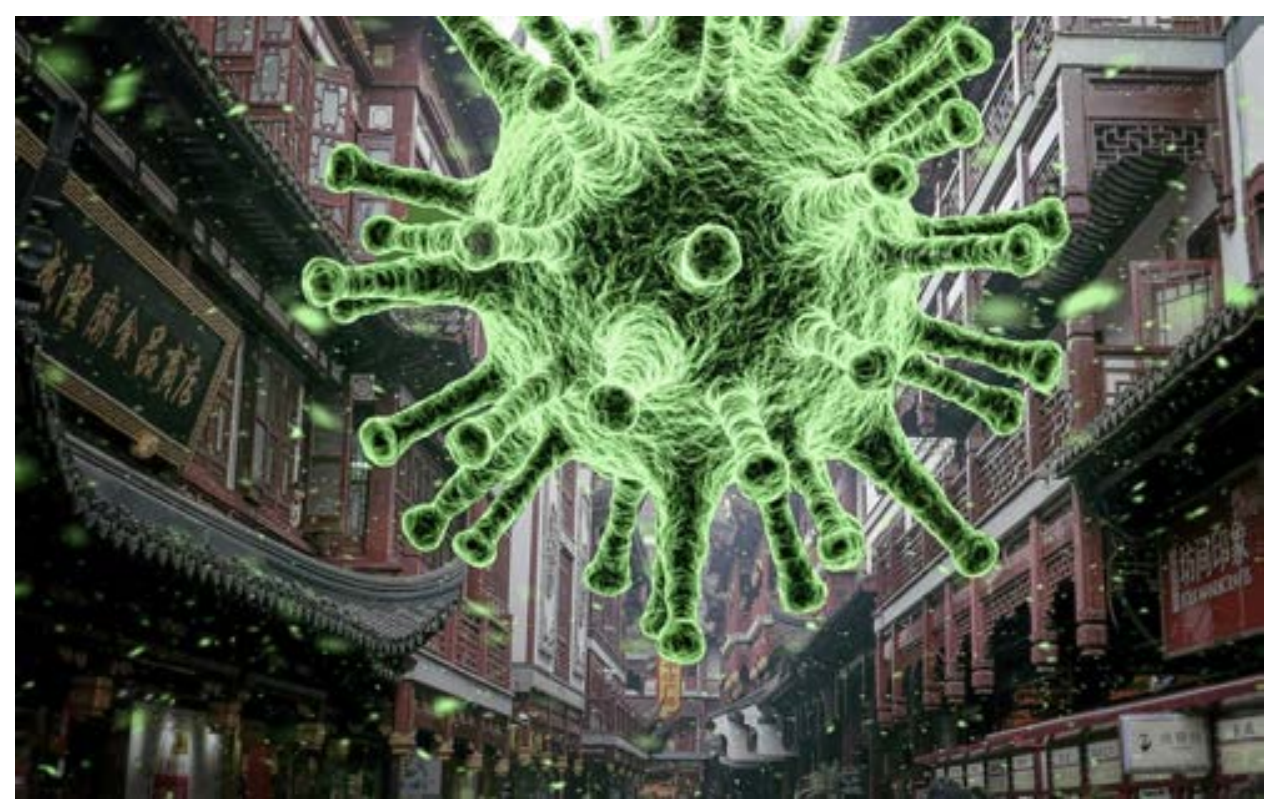


el primer caso importado en un paciente de 61 años residente de Wuhan. Estados Unidos de Norteamérica reportó su primer caso confirmado el 20 de enero en un paciente de 35 años que viajó a Wuhan. Entre el 23 y 24 de enero se inició la cuarentena en área metropolitana de la ciudad de Wuhan junto con varias ciudades cercanas y se establecieron medidas de control en los medios de transporte aéreos y terrestres nacionales e internacionales. ${ }^{10} \mathrm{La}$ OMS, el 30 de enero, declaró a la infección por nCoV-2019 una emergencia internacional de salud pública. ${ }^{11}$

El 11 de febrero el nombre de la enfermedad cambió oficialmente a COVID-19 (coronavirus disease). El nombre del virus, posterior al análisis genómico de las secuencias, es SARS-CoV-2.

Al 02 de marzo de 2020 se han confirmado 88,948 casos y 2,977 defunciones, con una tasa de letalidad global de $3.4 \%$, se reportan casos graves en $20 \%$. La epidemia se ha propagado a 64 países. ${ }^{12}$ El riesgo a nivel global es muy alto.

\section{Aspectos clínicos de infección por COVID-19}

El espectro clínico en los pacientes infectados por SARS-CoV-2 es amplio, incluye desde casos asintomáticos hasta neumonías graves e incluso la muerte. Las características clínicas de los primeros 425 casos confirmados tenían una mediana de edad de 59 años, con rangos en 15 a 89 años, $56 \%$ eran hombres, y en un inicio no se reportó ningún menor de 15 años. Antes del primero de enero $69 \%$ de los pacientes que presentaron neumonía tuvieron exposición a los mercados húmedos de Wuhan; sin embargo, en el transcurso de los días la asociación fue disminuyendo e incrementaron el número de casos con antecedentes de contactos con enfermos e incluso con personas asintomáticas, de igual forma se reportó un incremento de casos en personal de salud. ${ }^{6}$ Se demostró la transmisión de una persona a otra y se sabe que el mecanismo de transmisión es mediante gotas respiratorias y contacto cercano, la transmisión fecal-oral también se ha sugerido y recientemente se ha propuesto un mecanismo de transmisión vertical. ${ }^{13-15}$ Se estima un número básico de reproducción $R_{0}$ de 2.2 (95\% IC, 1.4 a 3.9), lo que nos indica que por cada persona infectada habrá transmisión a otras 2.2 personas (número similar al de la influenza). El periodo de incubación varía de dos a 21 días, con mayor frecuencia entre tres y siete días.

La información de mayor relevancia clínica se ha establecido con respecto a los pacientes que desarrollan neumonía: los primeros 99 casos confirmados tenían una edad promedio de 55 años, ningún menor de 15 años, y $51 \%$ de los pacientes presentaban una comorbilidad, las más frecuentes fueron enfermedades cardiovasculares y cerebrovasculares y 33\% presentó alguna complicación, principalmente síndrome de distrés respiratorio agudo. ${ }^{16}$ En otra serie de 138 pacientes hospitalizados con neumonía, se ingresaron 36 pacientes a terapia intensiva (26.1\%) por disfunción orgánica, en comparación con los pacientes que no ingresaron a terapia intensiva fueron significativamente mayores en edad (mediana, 66 años versus 51 años; $p<0.001$ ) con un tiempo medio desde el inicio de los síntomas hasta su ingreso a terapia intensiva de 10 días. ${ }^{17}$ Los síntomas que se han reportado en particular son fiebre, tos seca, disnea, mialgias y fatiga, menos frecuente confusión, cefalea, dolor faríngeo, rinorrea, dolor abdominal, diarrea, náuseas y vómitos. Con respecto a estudios de laboratorio se observan leucocitos en valores normales o bajos, linfopenia, elevación de enzimas hepáticas, y elevación de enzimas musculares. Las radiografías de tórax muestran afectación intersticial, más notable en el pulmón periférico. En el caso de las tomografías se define mejor la afectación pulmonar con imágenes en vidrio despulido y áreas de consolidación segmentarias en ambos pulmones, aunque un estudio de imagen normal no descarta la infección y hasta el momento se desconocen las secuelas imagenológicas a largo plazo. ${ }^{14,16,17}$

El diagnóstico se realiza mediante prueba RT-PCR de muestras respiratorias que incluyen hisopado orofaríngeo, nasofaríngeo, esputo, lavado broncoalveolar y aspirados traqueales. Para su recolección, almacenamiento y transporte se deben seguir las recomendaciones de la OMS, ${ }^{18} \mathrm{y}$ los lineamientos establecidos en cada país. ${ }^{19}$

En una epidemia es importante definir los grupos de riesgo de complicaciones por la infección, en el caso de infección por SARS-CoV-2 desde el inicio se observó mayor gravedad en pacientes de mayor edad y quienes presentaban una comorbilidad. Se conocen otros grupos de riesgo por la experiencia previa con otros coronavirus y por la información actual de los casos confirmados.

La infección por SARS-CoV y MERS-CoV demostró una alta transmisión nosocomial y al personal de salud, por lo que se considera un grupo de riesgo. El 25 de enero se enviaron trabajadores médicos a Wuhan para la atención de los pacientes afectados por COVID-2019 en el Hospital de Zhongnan, de los casos confirmados se sospecha que la transmisión hospitalaria es el mecanismo de infección probable al personal de salud y a pacientes hospitalizados en 29 y $12.3 \%$, 
respectivamente. ${ }^{17} \mathrm{La}$ OMS reportó el 14 de febrero 1,716 casos confirmados en trabajadores de la salud, que corresponden a $3.4 \%$ del total de casos, incluyendo seis defunciones. ${ }^{20}$ No sólo se ha identificado el riesgo de infección, sino también la respuesta de salud mental que se presenta ante una emergencia de salud pública, de tal forma que la Comisión Nacional de Salud en China, el 27 de enero, publicó la primera guía de intervención psicológica para el personal de salud en caso de crisis psicológica por COVID-19. ${ }^{21}$

En el caso de mujeres embarazadas aún no se conoce el potencial patogénico de la infección, pero por el comportamiento del SARS-CoV y MERS-CoV se cree que puedan tener mayor riesgo por complicaciones severas tanto maternas como perinatales. ${ }^{22}$ Un estudio que reportó las características clínicas de nueve mujeres embarazadas con neumonía por COVID-19 no encontró diferencias en comparación con no embarazadas, la resolución del embarazo en todos fue mediante cesárea y las pruebas realizadas en líquido amniótico, cordón umbilical, hisopado faríngeo de los recién nacidos y leche materna fueron negativas para SARS-CoV-2, la limitación principal fue el número de pacientes. ${ }^{23}$

$\mathrm{Al} 31$ de enero, en China, se notificaron 28 casos pediátricos con edades que oscilaban entre un mes y 17 años, generalmente presentan síntomas leves, algunos detectados como parte del escrutinio de estudio de contacto, con resolución de los síntomas en una a dos semanas, con buen pronóstico y de acuerdo con los casos reportados el contacto cercano con personas infectadas es la principal ruta de transmisión, hasta el momento no se ha registrado ninguna muerte en este grupo de edad. ${ }^{14} \mathrm{El} 05$ de febrero se confirmaron dos casos de recién nacidos con pruebas positivas para $\mathrm{nCoV}-2019$, el de menor edad con 36 horas de vida y al siguiente día se realizó un consenso de expertos sobre el manejo perinatal y neonatal para la prevención y control en este grupo de edad. ${ }^{15}$ En otro reporte de nueve niños infectados menores de un año también se describe el comportamiento leve de la enfermedad, todos se hospitalizaron, ninguno tuvo complicaciones o enfermedad grave. Todos tuvieron al menos un miembro de la familia que estaba infectado. ${ }^{24}$

Hasta la fecha no hay un tratamiento específico y probado contra la infección por COVID-2019, se recomienda de forma inmediata establecer las medidas de prevención y control, con una monitorización estrecha, aunque en los casos leves se puede dar manejo ambulatorio, en los casos que se requiera hospitalización se debe hacer en hospitales y áreas designadas para su manejo, con medidas de precaución estándar, de contacto por gotas y de vía aérea cuando se realicen procedimientos que generen aerosoles. Proporcionar tratamiento sintomático y de soporte respiratorio y en casos graves con disfunción orgánica su ingreso a terapia intensiva. No existen ensayos clínicos aleatorizados que apoyen un fármaco antiviral, las guías han propuesto la administración de alfa-interferón y el uso de lopinavir/ritonavir..$^{25,26}$ En Estados Unidos el primer caso reportado también fue el primero en utilizar como parte de su tratamiento remdesivir, que es un análogo de adenosina, y junto con la cloroquina han demostrado inhibir el SARS-CoV-2 in vitro. ${ }^{27}$

\section{Situación epidemiológica en México}

Desde que se dio a conocer el brote de COVID-2019, se establecieron medidas de control y para el 09 de enero de 2020, la Dirección General de Epidemiología emitió un aviso preventivo de viajes a China, y posteriormente se publicó el lineamiento estandarizado para la vigilancia epidemiológica y por laboratorio de enfermedad por nCoV-2019, en el que se establecieron las definiciones operacionales, las cuales se encuentran aún sujetas a cambio. Se considera un caso sospechoso una persona de cualquier edad que presente enfermedad respiratoria aguda y que cuente con el antecedente de viaje o estancia en países con transmisión local comunitaria: China, Hong Kong, Corea del Sur, Japón, Italia, Irán, Singapur, Francia, Alemania, España y Estados Unidos de América, o haber estado en contacto con un caso confirmado o bajo investigación hasta 14 días antes del inicio de los síntomas. Se considera un caso confirmado una persona que cumpla con definición operacional de caso sospechoso y confirmación por laboratorio emitido por el Instituto de Diagnóstico y Referencia Epidemiológico. ${ }^{19}$

El 27 de febrero de 2020 por la mañana se anunció un paciente que tuvo una prueba positiva. Este hombre acudió a consulta al INER, tenía el antecedente de haber viajado a Bergamo, Italia, donde estuvo en contacto con un individuo infectado. El 28 de febrero el INDRE confirmó que se trataba del primer caso de COVID-10 en México. En el seguimiento de estos casos se encontró a tres contactos que también viajaron a Italia. En la investigación epidemiológica se hizo seguimiento de los cuatro casos, tres presentaron síntomas leves y se confirmaron como casos de COVID-19. Dos pacientes permanecieron en la Ciudad de México y uno en Sinaloa. El cuarto sujeto no presentó síntomas y quedó como portador. El 29 de febrero de 2020, se reportó un caso positivo en una paciente de sexo femenino que 
regresó a Torreón, Coahuila, también procedente de Italia. Sus síntomas son leves y se quedó en casa para cuidados domiciliarios. Un quinto caso fue confirmado en Tuxtla Gutiérrez, Chiapas, es una joven de 18 años, con síntomas leves, que también llegó de Italia y es contacto del $4^{\circ}$ caso de Torreón, Coahuila. Al $1^{\circ}$ de marzo todos los casos en México son importados. Se ha realizado la investigación en 76 sujetos, de los cuales 60 son negativos, 11 están como sospechosos y cinco fueron confirmados. ${ }^{28}$

Hasta el momento no se cuenta con una vacuna para la prevención de la infección por COVID-2019, por lo que la mejor manera de prevenir la infección es evitar exponerse al virus. Por lo anterior, se enumerarán las medidas que han demostrado que evitan el contagio. ${ }^{29}$

1. Lavado frecuente de manos con agua y jabón al menos por 60 segundos, en especial después de ir al baño, antes de comer, y después de sonarse la nariz, toser o estornudar, así como después del contacto directo con personas enfermas o su entorno. En el caso de que las manos no estén visiblemente sucias o contaminadas, se puede utilizar un producto para desinfección de manos que contenga $70 \%$ de alcohol (por 20-30 segundos).

2. Limpiar y desinfectar los objetos y las superficies que se tocan con frecuencia, usando un producto común de limpieza de uso doméstico en rociador o toallita.

3. Cubrirse la nariz y la boca con un pañuelo desechable al toser o estornudar y depositarlo inmediatamente en la basura (de preferencia en un cesto manos libres para no tocar la tapa).

4. Evitar el contacto cercano con personas que padecen infecciones respiratorias agudas (mantenerse al menos a un metro de distancia, mayor a la longitud de un brazo).

5. Reducir el tiempo en lugares concurridos, así disminuye la probabilidad de estar en contacto con personas enfermas.

6. Evitar el contacto sin protección con animales de granja o animales salvajes. Después del contacto, lavarse las manos con agua y jabón.

7. Evitar tocarse la cara, sobre todo ojos y la boca.

8. Las personas con síntomas de infección respiratoria aguda (tos, rinorrea, dolor faríngeo, estornudos) deben practicar la etiqueta respiratoria, es decir, cubrirse la nariz y boca con un pañuelo desechable al toser y estornudar, si no se cuenta con un pañuelo utilizar el ángulo del codo.
9. Las personas con síntomas de infección respiratoria no deben acudir a lugares y espacios concurridos (cines, fiestas, conciertos, transporte público, entre otros).

10. Evitar saludar de mano y de beso. Las medidas se refuerzan de acuerdo a la situación existente.

11. Acudir al médico si se presenta una infección respiratoria con fiebre, cefalea, dolor faríngeo, rinorrea. No automedicarse. Es recomendable utilizar una mascarilla facial (cubrebocas) durante los traslados.

12. Acudir inmediatamente al médico si hay además falta de aire o dificultad respiratoria.

13. No se recomienda el uso rutinario de mascarilla facial en personas sanas.

14. Dentro de las instalaciones sanitarias, mejorar las prácticas de precaución estándar y por mecanismo de transmisión para prevención y control de infecciones.

\section{CONCLUSIONES}

La infección por COVID-19 ha afectado a 93 países con más de 100,000 casos y 3,486 muertes. Su comportamiento epidémico preocupa a nivel internacional, se ha generado un incremento en la evidencia científica con respecto a esta enfermedad aún con muchas interrogantes que responder. $\mathrm{Al}$ no contar en este momento con un tratamiento específico se debe continuar con las medidas de prevención y control recomendadas por la OMS.

\section{REFERENCIAS}

1. International Committee on Taxonomy of Viruses (ICTV). [Internet]. [Access 5 February of 2020] Available in: https://talk.ictvonline.org/ taxonomy/.

2. Su S, Wong G, Shi W, Liu J, Lai ACK, Zhou J et al. Epidemiology, genetic recombination, and pathogenesis of coronaviruses. Trends Microbiol. 2016; 24(6): 490-502. doi: 10.1016/j.tim.2016.03.003.

3. Song Z, Xu Y, Bao L, Zhang L, Yu P, Qu Y et al. From SARS to MERS, thrusting coronaviruses into the spotlight. Viruses. 2019; 11(1): 59. doi: 10.3390/v11010059.

4. Cui J, Li F, Shi Z-L. Origin and evolution of pathogenic coronaviruses. Nat Rev Microbiol. 2019; 17(3): 181-192. doi: 10.1038/s41579-018-0118-9.

5. Zumla A, Chan JFW, Azhar El, Hui DSC, Yuen KY. Coronavirusesdrug discovery and therapeutic options. Nat Rev Drug Discov. 2016; 15(5): 327-347. doi: 10.1038/nrd.2015.37.

6. Li Q, Guan X, Wu P, Wang X, Zhou L, Trong Y et al. Early transmission dynamics in wuhan, china, of novel coronavirusinfected pneumonia. N Engl J Med. 2020. doi: 10.1056/ NEJMoa2001316.

7. Zhu N, Zhang D, Wang W, Li X, Yang B, Song J. A novel coronavirus from patients with pneumonia in China, 2019. N Engl J Med. 2020. doi: 10.1056/NEJMoa2001017.

8. The 2019-nCoV Outbreak Joint Field Epidemiology Investigation 
Team, Li Q. An outbreak of NCIP (2019-nCoV) infection in China-Wuhan, Hubei Province, 2019-2020. China CDC Weekly. 2020; 2(5): 79-80. [Internet] [Access 08 February of 2020] Available in: http://weekly.chinacdc.cn/fileCCDCW/journal/article/ ccdcw/2020/5/PDF/NCIP.pdf.

9. Zhou P, Yang XL, Wang XG, Hu B, Zhang L, Zhang $W$ et al. A pneumonia outbreak associated with a new coronavirus of probable bat origin. Nature. 2020. [Internet]. Available in: https:// doi.org/10.1038/s41586-020-2012-7.

10. Wu JT, Leung K, Leung GM. Nowcasting and forecasting the potential domestic and international spread of the 2019-nCoV outbreak originating in Wuhan, China: a modelling study. Lancet [Internet]. 2020. Available in: https://www.thelancet.com/journals/ lancet/article/PIIS0140-6736(20)30260-9/fulltext.

11. OMS. Declaración sobre la segunda reunión del Comité de Emergencias del Reglamento Sanitario Internacional (2005) acerca del brote del nuevo coronavirus (2019-nCoV) [Internet]. (Fecha de acceso 8 de febrero de 2020) Disponible en: https:// www.who.int/es/news-room/detail/30-01-2020-statement-onthe-second-meeting-of-the-international-health-regulations(2005)-emergency-committee-regarding-the-outbreak-of-novelcoronavirus-(2019-ncov).

12. WHO Coronavirus disease 2019 (COVID-19). Situation report 42. https://www.who.int/emergencies/diseases/novelcoronavirus-2019/situation-reports (fecha de acceso 2 de marzo de 2020)

13. Fuk-Woo J, Yuan S, Kok KH, Kai-Wang T, Chu H, Yang J et al. A familial cluster of pneumonia associated with the 2019 novel coronavirus indicating person-to-person transmission: a study of a family cluster. Lancet. 2020; 395(10223): 514-523. doi: 10.1016/ S0140-6736(20)30154-9.

14. Shen K, Yang Y, Wang T, Zhao D, Jiang Y, Jin R et al. Diagnosis, treatment, and prevention of 2019 novel coronavirus infection in children: experts' consensus statement. World J Pediatr. 2020. doi: 10.1007/s12519-020-00343-7.

15. Wang L, Shi Y, Xiao T, Fu J, Feng X, Mu D et al. Chinese expert consensus on the perinatal and neonatal management for the prevention and control of the 2019 novel coronavirus infection (First edition). Ann Transl Med. 2020 [Internet]. [Access 8 February of 2019] Available in: http://dx.doi.org/10.21037/atm.2020.02.20.

16. Chen N, Zhou M, Dong X, Qu J, Gong F, Han Y et al. Epidemiological and clinical characteristics of 99 cases of 2019 novel coronavirus pneumonia in Wuhan, China: a descriptive study. Lancet. 2020; 395(10223): 507-513. doi: 10.1016/S01406736(20)30211-7.

17. Wang D, Hu B, Hu C, Zhu F, Liu X, Zhang J et al. Clinical Characteristics of 138 hospitalized patients with 2019 novel coronavirus-infected pneumonia in Wuhan, China. JAMA. 2020. doi: 10.1001/jama.2020.1585.

18. OMS Vigilancia Mundial de la infección humana por el nuevo coronavirus (2019-nCoV). Orientaciones provisionales. 31 de enero de 2020. [Internet]. [Fecha de acceso 8 de febrero 2019] Disponible en: who.int/es/emergencies/diseases/novelcoronavirus-2019/technical-guidance.

19. Lineamiento estandarizado para la Vigilancia Epidemiológica y por Laboratorio de Enfermedad por 2019-NCOV. Secretaría de Salud. Subsecretaría de Prevención y Promoción de la Salud. Dirección General de Epidemiología. 7 de febrero 2020. [Internet] [Fecha de acceso 8 de febrero 2020] Disponible en: www.gob. $\mathrm{mx} / \mathrm{salud}$.

20. OMS. Informe de situación de enfermedad por Coronavirus 2019 (COVID-19) $N^{\circ} 25$. [Internet] [Fecha de acceso 15 de febrero de 2020] Disponible en: https://www.who.int/docs/default-source/ coronaviruse/situation-reports/20200214-sitrep-25-covid-19. pdf?sfvrsn=61dda7d_2.

21. Kang L, Li Y, Hu S, Chen M, Yang C, Yang BX et al. The mental health of medical workers in Wuhan, China dealing with the 2019 novel coronavirus. Lancet Psychiatry. 2020 [Internet]. (Access 10 February of 2020) Available in: https://www.thelancet.com/journals/ lanpsy/article/PIIS2215-0366(20)30047-X.

22. Favre G, Pomar L, Musso D, Baud D. 2019-nCoV epidemic: what about pregnancies? Lancet. 2020. doi: 10.1016/S01406736(20)30311-1.

23. Chen H, Guo J, Wang C, Luo F, Yu X, Zhang W et al. Clinical characteristics and intrauterine vertical transmission potential of COVID-19 infection in nine pregnant women: a retrospective review of medical records. Lancet. [Internet]. [Access 15 February of 2020] Available in: https://doi.org/10.1016/S0140-6736(20)30360-3.

24. Wei M, Yuan J, Liu Y, Fu T, Yu X, Zhang Z. Novel coronavirus infection in hospitalized infants under 1 year of age in China. JAMA. Published online February 14, 2020. doi:10.1001/jama.2020.2131.

25. Jin YH, Cai L, Cheng ZS, Cheng H, Deng T, Fan YP et al. A rapid advice guideline for the diagnosis and treatment of 2019 novel coronavirus (2019-nCoV) infected pneumonia (standard version). Mil Med Res. 2020; 7(1): 4. [Internet]. Available in: https://doi. org/10.1186/s40779-020-0233-6.

26. Lu H. Drug treatment options for the 2019-new coronavirus (2019nCoV). Biosci Trends. 2020 doi: 10.5582/bst.2020.01020.

27. Wang M, Cao R, Zhang L, Yang X, Liu J, Xu M et al. Remdesivir and chloroquine effectively inhibit the recently emerged novel coronavirus (2019-nCoV) in vitro. Cell Res. 2020: 1-3. doi: 10.1038/ s41422-020-0282-0.

28. Comunicado Técnico diario. Nuevo Coronavirus en el mundo (COVID-19). Secretaría de Salud. https://www.gob.mx/cms/ uploads/attachment/file/538005/Comunicado_Tecnico_Diario_ COVID-19_2020.03.01.pdf (fecha de acceso 2 de marzo 2020)

29. WHO: Infection Prevention and Control During Health Care When Novel Coronavirus (nCoV) Infection Is Suspected: Interim Guidance. WHO website. Updated January 25, 2020. [Access 13 February of 2020] Available in: https://www.who.int/publicationsdetail/infection-prevention-and-control-during-health-care-whennovel-coronavirus-(ncov)-infection-is-suspected-20200125. 